\title{
APPLICATION OF VIRTUAL ENVIRONMENTS IN TRAINING OF ERGATIC SYSTEM OPERATORS
}

\author{
Igor Petukhov*, Lyudmila Steshina, Andrei Glazyrin \\ Volga State University of Technology, Russian Federation
}

The paper discusses the approach to the training of ergatic system operators, which implies the application of virtual reality elements. It presents the review of the existing models of the virtual environment application, offers a system model of the operator's response, systemizes the main structural and functional elements of personal experience in virtual environments.

Key words: Ergatic control systems, Human-operator, Virtual reality, Virtual environment

\section{INTRODUCTION}

Most of the contemporary technological objects can be classified as ergatic systems (ES). The main peculiarity of such systems is their sociopsychological aspects that make them different from typical man-machine systems. The modern technology causes the development of significantly altered ergatic functions. The implementation of virtual and alternate realities ensures the higher effectiveness of the ES operator training and professional activities. At the same time, their operational gnostic and practical functions alter, and their working practice transforms, too.

In this context, many issues of the arrangement of the ES operators' working practice in virtual environments are not yet understood, the optimization of the training process in virtual environments requires further discussions, the confident data on the efficiency of the operators trained with the application of a virtual content are missing.

The goal of this paper is to study the possibilities of virtual environments within the training of ES operating personnel.

\section{THEORETICAL ANALYSIS}

The virtual reality (VR) essentially differs from other software systems, first of all, by its strong psychophysiological impact on a person. It deals with a so-called presence effect or immersion into the environment [1] that is empirically proved, in particular, by physiological measurements: the body temperature, muscular tension, cardiovasculare cardiogram, etc. [2], and is determined by multimodal environmental characteristics [3,20].

It should be noted that the subjective sensation of immersion depends on many factors like concentration, imagination, proneness to immersion and detailed elaboration of images [4]. In addition, different components of immersion can make different impacts on the relevant types of tasks [5] and transfer the knowledge from the VR into the real environment [6].

Unfortunately, most of the current models consider the user as a secondary element of the system or do not consider them at all. The focus of the semantic profile moves on the detailed elaboration of the imitation process [7] or on the software or hardware [8].

It its turn, VR is able to reproduce complex communicative connections [9], to imitate an internal psychological state [10], to activate cognitive functions [11], reproduce professional practices [12].

However, beside a positive effect this technology has a negative effect either; for example, cognitive dissonance. This phenomenon appears in the person mind due to discrepancies between their previous experience and the present situation or due to logic inconsistency of the virtual reality and a physical object, according to L. Festinger. In this situation a key role belongs to the depth of immersion into the virtual reality and the level of human-machine interaction. At the same time, the implementation of operators' commands in the virtual reality has a certain difficulty [13]. In this connection, the study of interface interaction between an operator and a technical system in the virtual reality with the aim of creating a better effect of the physical presence and better control using adaptive interfaces is rather relevant at the moment.

Currently, ensuring situational awareness (SA) is considered as the main problem of the ergatic control system (ECS). Operators are able to make effective decisions providing a proper operating process only if they have a required situational awareness. $[14,15]$.

Therefore, there is a number of questions connected with the definition of the subjective experience of a person's presence in the environment, revealing key elements and categories of such presence, and developing a human-oriented model for effective training simulation, etc.

\section{THE REVIEW OF THE CURRENT MODELS OF VIRTUAL ENVIRONMENT APPLICATION}

In comparison with traditional software interfaces VR comprises a great variety of objects, interactive techniques, behavior scenarios, communicative practices, etc. All this causes problems in the virtual environment 


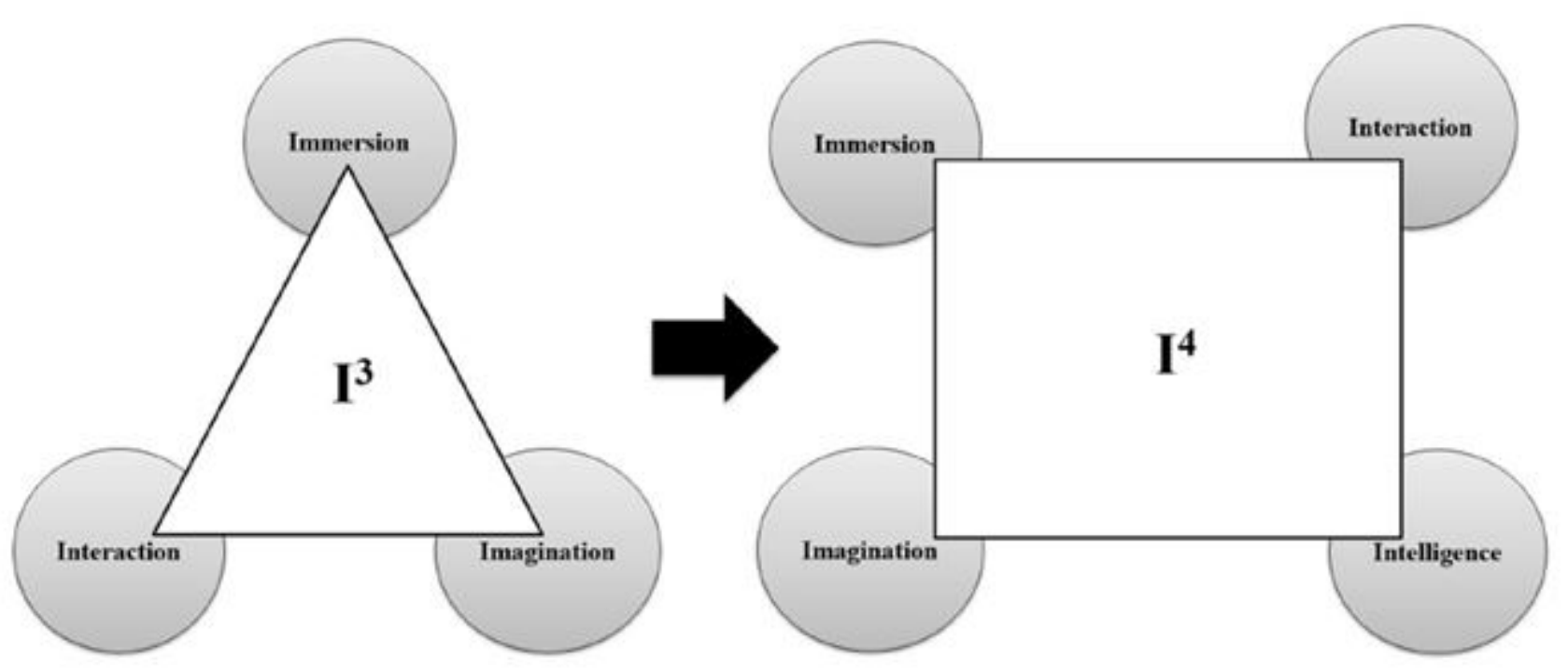

Figure 1: The 13 and 14 models [19]

modeling process that are related to the realism of the environment (design and implementation of the 3D content) [16], the co-existence of virtual and physical objects that can make an information exchange between each other [17], the interaction of the environment and a person (human characteristics, psychophysiological features, multimodal interaction) [18], etc.

In general, the VR model can be described through conceptual elements with semantic connections. In this context, the most representative model is I3 [19] that can be completed up to the 14 model shown in Figure 1.

It should be emphasized that generic categorization of conceptual elements cannot give a complete idea of the functioning and integration of the environment with the user. Also, there is neither division between a virtual environment, a technical system and a user nor defined two-sided communicative connections.

Another approach to the VR modeling defines the framework of the virtual environment that is characterized as external in relation to the user. This approach establishes

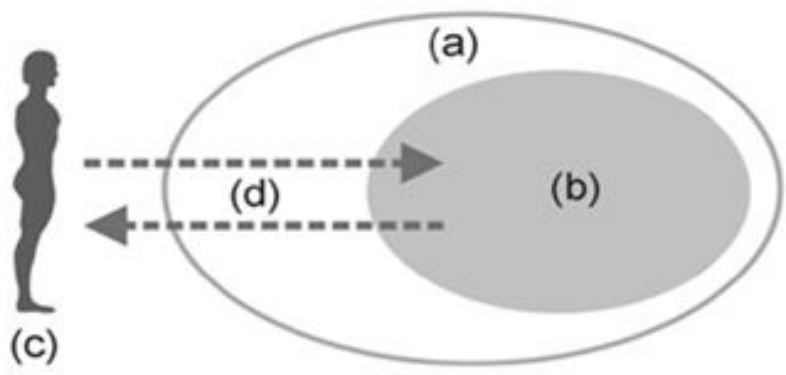

Figure 2: The simple diagram of the human-machine interaction, where (a) is a VR system, (b) is a virtual environment, (c) is a user, and (d) is interactive communication [16] a two-sided communicative connection between the user and VR. Therefore, the key difference is the introduction of a mediator that integrates the environment with the person. [16].

The VRID (Virtual Reality Interface Design) methodology presents a more formalized system of functional connections. This approach outlines the main functional levels of a virtual environment using technical and software decomposition. VRID deploys design templates, namely MVC (Model-View-Controller) [18].

According to this approach, the VR system can be described through three interconnected levels; (i) software; (ii) data input control; (iii) an interface (objects and data). On the other side, the VRID methodology renders the user as a source of inputs that come to the interaction module. There is no clear division between a virtual environment, technical equipment, and other elements. Semantic connections do not give a complete idea of the functional loading of a system. Therefore, we need a more focused approach to modeling, which could take into account a set of specific objectives being attained in the environment and present the detalization of the process.

One of the first attempts to outline and describe human and technical elements that establish independent spheres of interaction was made within the Conceptual VR Model (CVRM) [21].

This model represents the user as a complex system of input and output data processing. The emphasis is made on the functional loading of perceptive and muscular elements that provide the interaction with VR within the psychological (cognitive) circuit. The work efficiency in the environment depends on the individual ergonomics, work experience and sensibility to the virtual environment. CVRM moves the main focus of structuring the functional connections on the user and restricts the virtual environment by communication connections in the form of effectors and sensory responses. At the same 
time, this model does not take into consideration the objective sphere of the virtual environment application, where different scenarios and manipulations can be applied.

The abovementioned can help make preliminary conclusions on the criteria of modeling the virtual environment. The existing models do not represent the interaction process between a person and a virtual environment in full. They lack important factors of gaining subjective experience of the presence in the environment, which can make a great impact on the environment efficiency. We should take into account the simulation sphere of the environment application, which should comply to the intended use of the simulator and reproduce the specificity of training and simulated work in the virtual reality.

\section{THE MODEL OF A VIRTUAL ENVIRONMENT FOR THE TRAINING OF ERGATIC SYSTEM OPERATORS}

The analytical study of system models and the pathways of the operator's actions makes it possible to reveal the most significant stages and specificity of operator's functions that influence the efficiency of the operating activity through:

- accepting and perceiving information (the initial stage of receiving information from different sensory sources) [22];

- $\quad$ evaluating and processing information (finding out the most important information and, then, its analysis and evaluation) [23];

- $\quad$ making a decision (planning a certain activity) [24];

- implementing the decision made (physical movement) [24].

One of the key elements of different models of operator's responses is reasoning or conclusion that make the ground for making a final decision [25, 26]. Making a final decision is based on the analysis of individual facts of workings, which are transformed into an integrated pattern of the workings [27].
Therefore, it is essential to include a mechanism of accumulation of priority information to the degree when we get the possibility to compare this information with some familiar situation or model of an operator's behavior. It is necessary to give a detailed description of the cognitive and motor levels and to clarify the control circuit in the model of operator's responses.

According to the proposed model shown in Figure 3, an operator receives information through sensory canals and by means of a sensory analysis forms a situational model prototype (SMP). Then, an access to the Conceptual Models (CM) database (DB), which has been formed by an operator earlier, and a content-addressable search are performed using key attributes of the situational model. It should be noted that DB comprises gained experience (mental templates) of planning and responding to different situations of the operating activity.

This process fills 'gaps' of the situational model and it is converted into an integral situational model (ISM) which implies a description of the current situation, a forecast for its development, generation of response variants, an analysis and selection of a response variant in accordance with the current criteria. A newly-formed integral situational model (ISM) is added to the Conceptual Models database for the further usage. Then, a scheme of motor response (CMR) is created using a set of motor programs included into the database, and a motor program (MP) is generated and implemented. Further, this cycle is repeated.

Thus, the proposed system model accumulates the information flow of the background knowledge at the cognitive level. If there is 'enough' information for making certain decisions, such information is instantiated in ISM. In this case we can create a simulation environment that would take into account ISM and the process of extracting background knowledge and compare them with the real situation. Therefore, the reproduction of subjective perception of the professional environment is seemed to be of a higher priority than the copying of a working scenario without consideration of an operator's internal state.

STAGES OF OPERATOR'S ACTIVITY IN ERGATIC SYSTEM

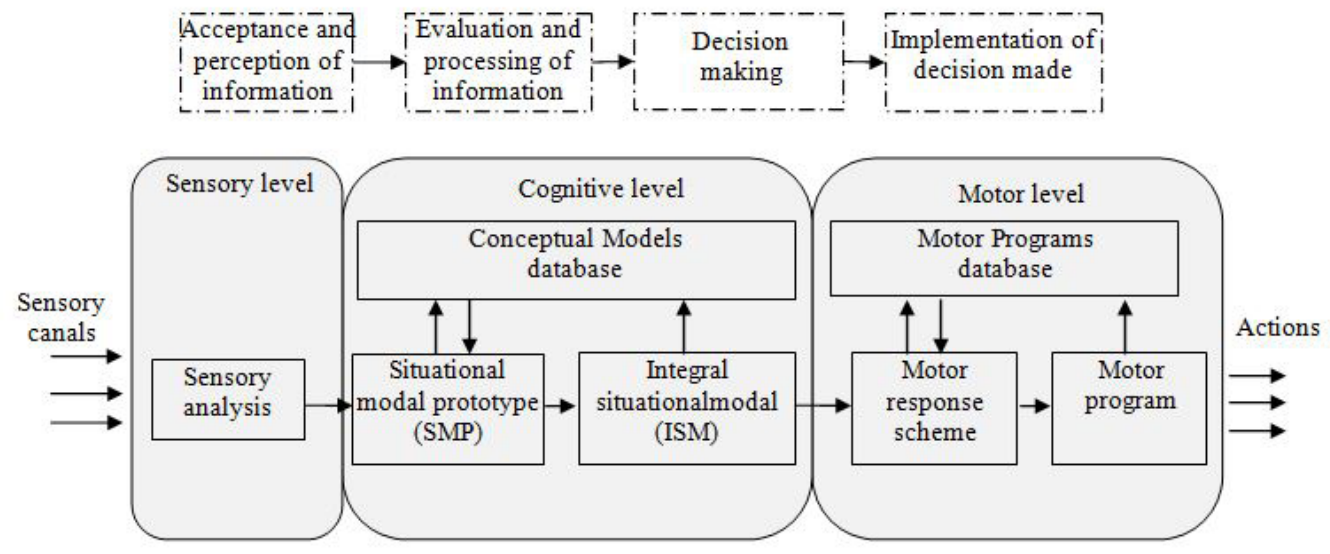

Figure 3: Thesystemmodelofan operators' response 
Subjective experience of the presence and actions in the virtual environment depends on numerous conditions.

K. Stanney et al. [3] state that the efficiency of the user in the virtual environments is, first of all, affected by the human factor. The main emphasis is made on psychic movements, human perception, multimodal interaction, parameters of objectives connected with mental processes.

D. Boyd [25] describes the connection between the efficiency of the virtual environment and the individual rience of the VR application for research into effective human visual perception in the real life [6].

N. Pares and R. Pares [16] define the human factor using the concept of virtual subjectiveness that determines the effectiveness of different objective attainment and the design of the virtual environment.

The essential element of the perception of a virtual environment is its representation as the latter establishes the balance between the physical equipment and the media content at the level of the reproduction of human metaphors and the recognition of cognitive patterns most often used in the everyday practice. The probability of the recognition of cognitive patterns depends on the confidence level of the representation of the virtual environment presented by its attribute groups shown in Table 1 .

The breach or incorrect transfer of the patterns may affect the environment efficiency in part of realism and generation of the psychophysiological state of a trainee.

The first group of attributes (spatial attributes) allow the operator to orient in the space and receive information on the controlled objects, their location, speed and the distance.

Beside the spatial parameters there are principles of physics and physical phenomena that determine the dynamic state of certain objects and the environment in general. If the spatial attributes mostly help orient in the environment, than the physical ones help perform certain subjective experience and, in addition, the proven expe-

actions with objects and the environment. This results in the evaluation of the situation and making certain decisions. In addition, the physical elements have the potential of a subjective psychophysiological impact and are often used for the simulation of hazardous environments.

The object simulating attributes of the representation specificate the certain object simulating activity and draw the user's attention to it with the aim of effective training; they build the main logic of training, namely: succession of actions, accuracy of manipulations, detailed visualization of the objective sphere, a possibility of a flexible control over the situation.

The emotional and psychological attributes help influence the emotional state of the user and simulate their behavior in emergency situations, in the rush and monotonous work modes.

The social grouping attributes ensure the interaction of users in the virtual environment, possibilities of their communication, group work, and in fact are determinative for the ergatic system.

The variation of priorities of representation attributes gives the opportunity to simulate a wide range of ergatic systems in the virtual environments with emphases on different objectives depending on the targeted function.

\section{CONCLUSION}

Application of virtual environments for the training of ES operators has its distinct advantages. First of all, they are associated with the reduced cost of training, the diversity of cognitive patterns of response and motor programs for emergency work modes. On the other side, application of virtual environments has a certain risk connected with inadequate responses.

According to the proposed system model of the operator's response, the confidence level of the conceptual model (the correspondence of the ES state to the reality) formed by the operator in the virtual environment is provided through the confidence level of the formation of representation attributes.

Table 1.

\begin{tabular}{|c|c|}
\hline Representation attribute & Description \\
\hline Spatial & $\begin{array}{l}\text { the distance and scale, sizes of objects, speed and movement of objects } \\
\text { (of the virtual content) }\end{array}$ \\
\hline Physical & $\begin{array}{l}\text { reproduction of principles of physics and physical phenomena similar to natural, } \\
\text { simulation of time intervals }\end{array}$ \\
\hline Object simulating & $\begin{array}{l}\text { simulation of the certain practical activity connected with the training process and } \\
\text { simulation activity details }\end{array}$ \\
\hline Emotional and psychological & $\begin{array}{l}\text { detalization of environment parameters connected with the influence on emotional } \\
\text { distress or phobic reactions }\end{array}$ \\
\hline Social grouping & $\begin{array}{l}\text { formation of the environment of the collaborative group activity of operators } \\
\text { (users) in the virtual environment, communicative aspects }\end{array}$ \\
\hline
\end{tabular}


In this context, we can control these attributes in dependence of the target function of training, for example, for simulation of group interaction the social grouping and object simulating attributes are of the highest priority.

The development of the model of representation that takes into account all attributes of representation is a promising direction for research and, at the same time, involves the greatest difficulty and scientific interest. This model determines a set of objective and psychological references that should synchronize the situational model prototype (SMP) and integral situational model (ISM).

The next level objective is the development of the models of interaction between the user and the technical system in the virtual environment, the operator's gaining subjective experience in the virtual environments and development of the conceptual model describing a current state of the ergatic system.

The research outcomes are obtained by the support of RF Ministry of Education and Science grant No. 25.1095.2017/4.6.

\section{REFERENCES}

1. Dinh H. Q. et al., (1999). Evaluating the importance of multi-sensory input on memory and the sense of presence in virtual environments. U: VirtualReality 1999, IEEE. 222-228.

2. Mestre D.R., \& Fuchs P., (2006). Immersion etprésence. U Fuchs P, Moreau G, Berthoz A, \&Vercher JL (Ur.), Le traité de la réalitévirtuelle. Paris: EcoledesMinesdeParis 309-38.

3. Stanney K.M., Mourant R. R., , \& Kennedy R. S., (1998). Human factors issues in virtual environments: A review of the literature. Presence: TeleoperatorsandVirtualEnvironments, 7(4), 327-351.

4. Laha B., \& Bowman D., (2012). Identifying the benefits of immersion in virtual reality for volume data visualization. U: Immersive visualization revisited workshop of the IEEE VR conference. 1-2.

5. Bailey J, Bailenson JN, Won AS, ,\& et al., (2012). Presence and memory: immersive virtual reality effects on cued recall. U: The International Society for Presence Research Annual Conference, Philadelphia, Pennsylvania. 24-26.

6. Davis, A. (2015). Virtual Reality Simulation: An Innovative Teaching Tool for Dietetics Experiential Education. TheOpenNutritionJournal, 9 (Suppl 1-M8), 65-75.

7. Aggarwal R. et al., (2007). Proving the effectiveness of virtual reality simulation for training in laparoscopic surgery. Annalsofsurgery, 246(5), 771-779.

8. Psotka J., (1995). Immersive training systems: Virtual reality and education and training. Instructionalscience, 23(8), 405-431.

9. Prasolova-Førland, E., Fominykh, M., Darisiro, R., Mørch, A.I., \& Hansen, D. (2014). Preparing for In- ternational Operations and Developing Scenarios for Inter-cultural Communication in a Cyberworld: A Norwegian Army Example. U: LectureNotesinComputerScience. 118-138.

10. Cha M. et al., (2012). A virtual reality based fire training simulator integrated with fire dynamics data. FireSafetyJournal, 50, 12-24.

11. Hanson K., \& Shelton B. E., (2008). Design and Development of Virtual Reality: Analysis of Challenges Faced by Educators. EducationalTechnology\&Society, 11(1), 118-131.

12. Wang P. et al., (2005). Designing a virtual reality simulator for neurosurgery. U: The seventh international conference on virtual reality, Laval, France. 19-22.

13. Gribova V.V., Kleschev A.S., ,Krylov D.A., , MoskalenkoPh. M., , Timchenko V.A., , Fedorischev L.A., \&Shalfeyeva E.A., (2016). The Base Technology for Intelligent Services Development with the Use of IACPaaS Cloud Platform. Part 3. An Interface Development and an Example of Applied Services Creation..SoftwareEngineering, 7(3), 99-107.

14. Endsley M. R., (1995). Toward a theory of situation awareness in dynamic systems. Human Factors: The Journal of the Human Factors and Ergonomics Society, 37(1), 32-64.

15. Endsley M. R. \& Jones W., (2013). Situation awareness. $U$ The Oxford Handbook of Cognitive Engineering. (str. 88-108).

16. Parés N.,\&Parés R., (2006). Towards a model for a virtual reality experience: the virtual subjectiveness. Presence: TeleoperatorsandVirtualEnvironments, 15(5), 524-538. doi:10.1162/pres.15.5.524

17. Cakmak H, \&Kuhnapfel U., (2000). Animation and simulation techniques for vrtraining systems in endoscopic surgery. U: EurographicsWorkshoponAnimationandSimulation. 173-185.

18. Tanriverdi V., \& Jacob R. J. K., (2001). VRID: a design model and methodology for developing virtual reality interfaces. U: The ACM symposium on Virtual reality software and technology. 175-182.

19. Al-Ahmari A. M. et al., (2016). Development of a virtual manufacturing assembly simulation system. AdvancesinMechanicalEngineering, 8(3), 1-13. doi:10.1177/1687814016639824

20. Burdea G., Richard P., \&Coiffet P., (1996). Multimodal virtual reality: Input-output devices, system integration, and human factors. InternationalJournalofHuman-Computerlnteraction, 8(1), 5-24.

21. Latta J. N., Oberg, D. J. (1994). Conceptual virtual reality model. IEEE Computer Graphics and Applications, 14, 23-29.

22. Petukhov I., (2011). Temporal Aspects of Human-machine Interaction in the Perception of Visual Information. U: ACHI 2011, The Fourth International Conference on Advances in Computer-Human Interactions, 
Gosier, Guadeloupe, France. 43-47.

23. Petukhov, I.V.,Rozhentsov, V.V., \&Aliev, M.T. (2007). On the accuracy of evaluations of temporal characteristics of visual perception. Bulletin of Experimental Biology and Medicine, 144(2), 267-268.

24. Petukhov I., Steshina L., Kurasov P., \&Tanryverdiev I., (2016). Decision Support System for Assessment of Vocational Aptitude of Man-machine Systems Operators. U: IEEE 8th International Conference on Intelligent Systems (IS'16). Sofia, Bulgaria.778-784.

25. Nilsson D., \&Kinateder M., (2015). Virtual Rality Experiments-The Future or a Dead End. U: 6th InternationalSymposiumHumanBehaviourinFire, Cambridge. 13-22.
26. Whitworth B. (2010).Simulating space and time // arXiv preprint arXiv:1011.5499.

27. Boyd D. (2000).Depth cues in virtual reality and real world //Department of Computer Science. Providence, Rhode Island, Brown University. Vol. 26. 